\title{
Editorial
}

\section{Second harmonic imaging: a new tune for an old fiddle?}

As with so many important discoveries in science, the significant improvements in echocardiographic image quality generated by second harmonic imaging were discovered almost by chance. Sound signals, including ultrasound transmitted through tissue, usually contain harmonics. These are additional frequencies, at multiples of the main frequency (called the fundamental). Therefore, second harmonic signals have been present within transmitted and reflected ultrasound data since echocardiography began. However, our ultrasound scanners have been "tuned in" to receive only the main or fundamental frequency and the second harmonic has been ignored. The paper by Franke and colleagues in this issue of Heart is one of several studies $^{1-6}$ that have appeared over the past year or so indicating the improved endocardial definition and therefore diagnostic accuracy obtained by using harmonic imaging. This improvement is especially important during stress echocardiography studies.

Second harmonic imaging was originally developed as a technique to increase the sensitivity of detection of ultrasound contrast agents, ${ }^{7-9}$ particularly when evaluating organ perfusion or to delineate cardiac chambers when assessing left ventricular function in patients with poor echocardiography windows. Ultrasound contrast agents usually consist of very small gas microspheres. They are strong reflectors of ultrasound and resonate when placed in an ultrasound field. As they resonate, they generate harmonics and these harmonic echoes are larger in amplitude than those from tissue. This makes it easier to detect and image the contrast agent within tissues and the cardiac chambers. However, it was soon appreciated that quality of the echocardiography image and endocardial definition was improved when second harmonic imaging was used alone, before the injection of any contrast agent.

There appear to be several factors that explain why second harmonic imaging improves echocardiography image quality and these rely on the fact that second harmonic frequencies are generated as the ultrasound pulse travels through tissue. Strictly speaking, a sound wave travelling through tissue should only contain the transmitted (fundamental) frequencies. However, a sound wave is rather like an ocean wave travelling towards the shore. As the wave approaches the shore it changes shape because the peak of the wave travels a little faster than the trough. When the
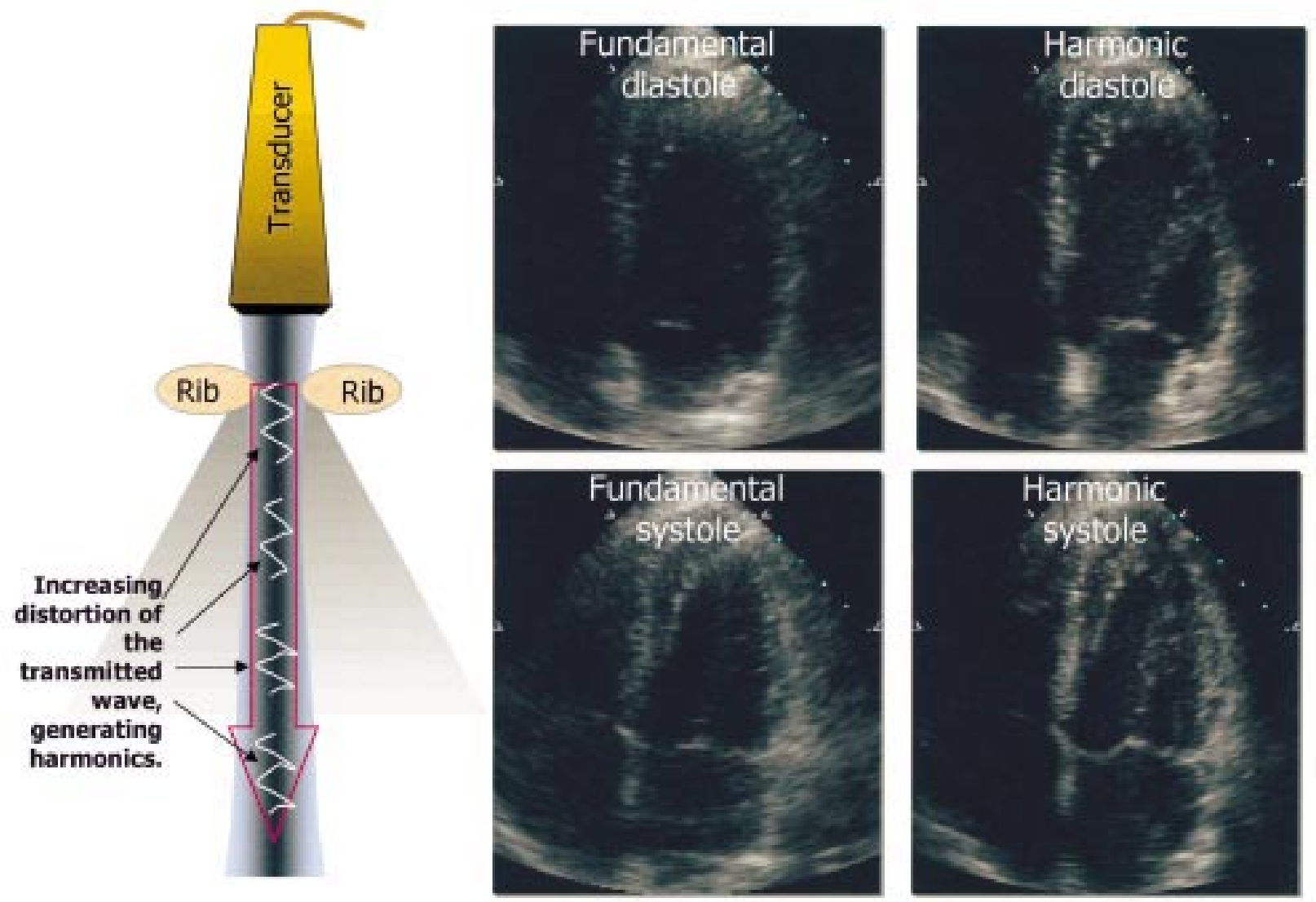

Figure 1 (Left) Diagram illustrating how, when second harmonic frequency imaging is used, image quality may be improved by narrowing the beam profile (enhancing lateral resolution) and by generation of increasing harmonics with depth, avoiding rib reverberation artefacts. (Right) Example of diastolic (upper) and systolic (lower) apical four chamber images using fundamental (left) and second harmonic imaging (right) in a patient with a suboptimal echocardiography window. Improvement in endocardial definition is seen especially in the diastolic image where the apex, lateral wall, and papillary muscle are better defined. In addition, overall image quality is improved by a reduction in clutter artefact caused by rib reverberations. 
wave breaks onto the shore, its peak has completely overtaken the trough and the wave shape has become distorted. An ultrasound wave travelling through tissue becomes distorted in an analogous way (fig 1). This distortion of the wave shape effectively generates additional sound frequencies, which are harmonics of the original (fundamental) frequency. The further the sound wave travels through tissue, the more it is distorted and more harmonics are produced. When the ultrasound system is operating in second harmonic mode, it is only these generated harmonics that are received and the fundamental signal is filtered out. As more harmonics are generated with increasing tissue depth, this acts as a form of depth compensation, effectively boosting the signal from deeper structures.

Many of the artefacts and distortions that occur in an echocardiogram are as a result of reverberations between the transducer and ribs. With second harmonic imaging, the harmonic signal is only generated after the sound wave has travelled a certain distance and this is usually beyond the rib cage, thereby avoiding the reverberation artefacts.

The harmonic signal is only generated in the centre of the transmitted ultrasound beam, because relatively high signal strength is required to create harmonics. This effectively means that the beam profile is kept narrower than for fundamental imaging. A narrow beam is important for high resolution images, because it reduces artefacts generated by intracardiac structures positioned near to the beam. Figure 1 illustrates diagrammatically the impact that second harmonic imaging has on the effective ultrasound beam profile and reduction in rib reverberation artefacts, which leads to these improvements in quality.

There is no doubt that this technology provides significant improvement in image quality, especially in patients with suboptimal echocardiography windows (fig 1). In most institutions, it has become the standard technique during stress echocardiography, because there is a critical need for good endocardial definition in this setting. During stress studies image quality often becomes suboptimal, because of respiratory artefacts and tachycardia. As we are often looking for very subtle changes in wall motion and thickening, high resolution images with good endocardial definition is vital. Harmonic imaging is also used routinely in most conventional transthoracic echocardiography studies in our own hospital, especially in adult patients where the echocardiography window is limited. I could not recommend purchasing a new ultrasound system that does not contain tissue harmonic imaging and indeed it may be worth upgrading to this facility, particularly if stress studies are to be undertaken.

Are there any disadvantages to using harmonic imaging? As a relatively high power ultrasound signal is required to generate harmonics it should be recognised that output levels are higher than with fundamental imaging, although still within regulated safety levels. As the harmonic signal generated within the tissues is of a higher frequency than the fundamental, depth penetration may be more limited. Therefore when visualising very deep structures, harmonic imaging may be limited in value. Finally, the type of processing required to filter out the received harmonic signal does make structures within the heart appear slightly thicker. For example, mitral valve leaflet thickness has been shown to be greater when visualised with harmonic as opposed to fundamental imaging. ${ }^{10}$ This may be important when assessing myxomatous degeneration in a floppy mitral valve or leaflet calcification. For the same reason, myocardial texture also appears different with second harmonic imaging. Clearly, it is necessary to develop a slightly different "normal echocardiography template" within the brain when interpreting harmonic echocardiography images.

In conclusion, it seems that one of the most dramatic improvements in echocardiographic image quality was discovered by chance and has in theory been available since the beginning of ultrasound imaging, given that second harmonic signals are automatically generated during tissue transmission of ultrasound. All we needed to do was to listen for this new tune from our old fiddle!

MARK J MONAGHAN

Consultant Clinical Scientist,

Care Group Director, King's College Hospital,

Denmark Hill, London SE5 9RS, UK

email:monaghan@compuserve.com

1 Franke A, Hoffmann R, Kühl HP, et al. Non-contrast second harmonic imaging improves interobserver agreement and accuracy of dobutamine stress echocardiography in patients with impaired image quality. Heart 2000;83:133-40

2 Caidahl K, Kazzam E, Lidberg J, et al. New concepts in echocardiography: harmonic imaging of tissue without contrast agents. Lancet 1998;352: $1264-70$.

3 Kornbluth M, Liang DH, Paloma A, et al. Native tissue harmonic imaging improves endocardial border definition and visualisation of cardiac structures. I Am Soc Echocardiogr 1998;11:693-701.

4 Spencer KT, Bednarz J, Rafter PG, et al. Use of harmonic imaging without echocardiographic contrast to improve two-dimensional image quality. $\mathrm{Am}$ 7 Cardiol 1998;82:794-9.

5 Becher $\mathrm{H}$, Tiemann K, Schlosser T, et al. Improvement in endocardial border delineation using tissue harmonic imaging. Echocardiography 1998;15: 511-16.

6 Chin D, Hancock J, Brown A, et al. Improved endocardial definition and evaluation of dobutamine stress echocardiography using second harmonic evaluation of dobutamine stress echocardiography

7 Monaghan MJ, Metcalfe JM, Odunlami S, et al. Digital radiofrequency echocardiography in the detection of myocardial contrast following intravenous administration of albunex. Eur Heart f 1993:14:1200-9.

8 de Jong N, Ten Cate FJ. New ultrasound contrast agents and technological innovations. Ultrasonics 1996;34:587-90.

9 Hancock J, Dittrich H, Jewitt DE, et al. Evaluation of myocardial, hepatic and renal perfusion in a variety of clinical conditions using an intravenous ultrasound contrast agent (Optison) and second harmonic imaging. Heart 1999;81:636-41

10 Norton M, Zaglavera T, Schuster J, et al. Increased apparent valve thickness with tissue harmonic imaging [abstract]. Heart 1998;80:P38. 